\title{
The Problem with Gout Is That It's Still Such a Problem
}
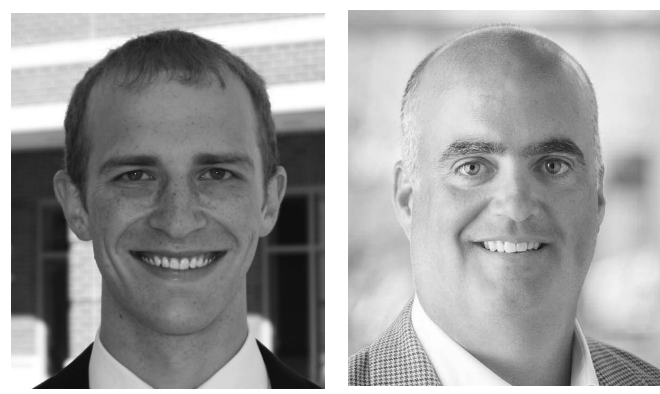

The problem at this point is that there is a problem.

$$
\text { - Captain Obvious }
$$

Gout is a problem. It's a big problem for patients, for caregivers and employers, and for healthcare systems characterized by dwindling resources, and it's a burgeoning problem for society at large. By far the most common form of inflammatory arthritis, gout affects about $4 \%$ of US adults ${ }^{1}$ and leads to substantial healthcare use among affected patients ${ }^{2}$. Gout's footprint is all the more relevant with its resulting morbidity, loss of productivity, and accelerated mortality experienced by its patients ${ }^{3,4}$. Two separate reports appearing in the current issue of The Journal examine emergency department (ED) use in gout care and serve to further highlight the burden of this ancient disease ${ }^{5,6}$.

Both these studies examine ED use in gout care using the National Emergency Department Sample (NEDS), an element of the Healthcare Cost and Utilization Project sponsored by the US Agency for Healthcare Research and Quality ${ }^{7}$. Highly generalizable and publically available, NEDS captures and characterizes 20\% of such visits occurring annually across the United States, and allows for national estimates of ED visits. Using NEDS data from 2006 through 2008, our group estimated that there were more than $170,000 \mathrm{ED}$ visits for gout as a primary diagnosis in 2008 alone, accounting for $\sim 0.2 \%$ of all ED visits nationally and generating corresponding total charges of more than $\$ 160$ million in a single year ${ }^{2}$. Extending this observation period, Jinno and colleagues note that between 2006 and 2012, ED visits for gout increased significantly by $14 \%$ overall, with an even greater increase observed for patients between 45 and 54 years of age ${ }^{5}$. Moreover, the authors note that resulting healthcare charges grew at an even higher rate, nearly doubling from \$156 million in 2006 to \$281 million in 2012. In a separate report, Singh and Yu found that nearly $8 \%$ of all ED visits for gout result in hospitalization, with a median inpatient stay approaching 3 days $^{6}$. For those with a primary gout diagnosis leading to hospitalization, total combined charges resulting from the ED and subsequent inpatient stay approached $\$ 350$ million. Recognizing the frequency of concomitant illnesses such as hypertension, diabetes, and renal failure in gout, Singh and Yu also examined the association of select comorbidities with the risk of hospitalization as well as generated charges. Perhaps not surprisingly, renal failure was strongly and adversely associated with both outcomes. Gout patients with renal failure were 8 times more likely to be hospitalized than gout patients without renal failure, even after accounting for other relevant patient- and hospital-level factors. Likewise, gout patients with renal failure accrued ED charges that on average were $\$ 647$ higher than gout patients without renal failure. These results suggest that concomitant renal disease, and to a lesser degree other comorbid illnesses, might identify the patients most likely to benefit from interventions aimed at reducing the growing burden of gout.

So gout remains a problem, one that does not appear to be going away soon, particularly for the nearly 1 of every 2 gout patients with concomitant moderate to severe chronic kidney disease ${ }^{8}$.

This may come as a surprise to many because the pathogenesis of gout (certainly in contrast to many other rheumatic conditions) is well understood; and the available longterm treatments are generally considered to be highly efficacious, well tolerated, and relatively inexpensive. As reported in other chronic diseases, ED use might serve as a bellwether of suboptimal healthcare delivery, reflecting poor access to care or the delivery of low-quality care (or both) for conditions that are otherwise preventable or typically nonurgent $^{9,10}$. This appears to be the case in gout. With rare exception, gout is highly treatable to the point that currently available urate lowering therapies, coupled with appropriately administered antiinflammatory prophylaxis, are said to offer "remission" or even a "cure," words rarely used to describe chronic disease. This supposition stands in stark contrast to reality. The fact remains that for most patients

See Trends in ED visits, page 1589; and Time trends, predictors, and outcomes, page 1581

Personal non-commercial use only. The Journal of Rheumatology Copyright (C) 2016. All rights reserved. 
with gout, optimal disease management and improved outcomes remain inexplicably elusive ${ }^{11,12}$. In a recent study of more than 13,000 patients with gout initiating allopurinol, less than one-third ever achieved target serum urate goals over an extended followup period ${ }^{8}$. Low goal achievement in this study appeared to be multifactorial and was associated with both patient and provider factors studied. Of the many factors examined, treatment adherence demonstrated the strongest association with achieving serum urate goals. Unfortunately, less than half of patients were adherent to their allopurinol treatment, a problem in gout care that has been highlighted by others ${ }^{13}$. In contrast to practices promulgated by international guideline efforts ${ }^{14}$, the vast majority of patients receiving new prescriptions for gout never underwent allopurinol dose escalation and almost $20 \%$ were given a single prescription that was never refilled ${ }^{8}$.

Echoing the refrain of Jinno and colleagues ${ }^{5}$, a perfect storm of rising disease incidence, increased costs, suboptimal healthcare quality, and poor patient outcomes together "reinforce the urgent need to improve gout management." But how do we most effectively improve healthcare quality for gout patients while simultaneously ensuring that those improvement efforts are cost-effective, sustainable, and widely implemented?

The "Chronic Care Model," developed by Wagner nearly 20 years ago, provides an initial roadmap ${ }^{15}$. The model was developed in response to the growing recognition that patients, healthcare providers, and the healthcare system are too often ill prepared or ill equipped to address chronic care needs. According to this model, there are 6 areas where interventions might be focused to improve chronic care, such as that needed for effective gout management: (1) community resources and policies, (2) self-management support, (3) delivery system design, (4) decision support, (5) clinical information systems, and (6) the organization of healthcare. Ultimately, targeting these areas has the potential to facilitate "an informed, activated patient interacting with a prepared, proactive practice team" to produce "high-quality, satisfying encounters and improved outcomes." Data strongly suggest that these areas are not optimally leveraged at present, because patients are not fully informed and providers do not appear to be adequately prepared or sufficiently proactive. Despite demonstrating relatively high levels of other gout-specific knowledge, only a small proportion of allopurinol-treated patients (14\% in a recent study by our group) can identify the target serum urate concentration used to guide urate-lowering treatment ${ }^{16}$. Sharing with patients the concept of uric acid as "the longterm treatment target," as the gout management guidelines suggest ${ }^{14}$, may be an important and underused conceptual anchor to improve patient engagement in self-management activities including adherence to gout treatment. Likewise, provider practices in gout management are all too often best characterized by clinical inertia, particularly in the widespread failure of providers to intensify urate-lowering treatment when it is clearly indicated ${ }^{8}$.

Gout remains a problem, but there may be a path forward. Preliminary reports suggest that the problems of suboptimal medication adherence and clinical inertia in the escalation of urate-lowering therapy can be overcome through patient engagement and system redesign. In a limited proof-of-concept, single-center study, the deployment of a comprehensive nurse-led intervention consisting of patient education, individualized lifestyle advice, and appropriate pharmacological management resulted in more than $90 \%$ of patients with gout achieving serum urate goals after 1 year of treatment ${ }^{17}$. For other medical conditions, the integration of technology has been used to optimize patient-provider communications and to improve the effect of similar healthcare interventions. For instance, a pharmacist-managed anticoagulation clinic used a centralized telephone system to engage patients directly and reduce anticoagulation therapy-related complications by nearly $40 \%$ relative to usual care $^{18}$. To date, however, there have been no randomized controlled studies examining such an intervention in gout. Recognizing this important evidence gap, we have recently embarked on a large multidisciplinary effort with the goal of improving treatment adherence and outcomes among gout patients initiating urate-lowering therapy across a single integrated health system. Using a large pragmatic cluster-randomized trial design, we are actively comparing a scalable and highly automated pharmacist-led intervention to usual care with a primary study endpoint of achieving serum urate goals at 1 year ${ }^{19}$.

Whether this intervention or others to follow result in meaningful or sustainable improvements in gout management on a large scale remains to be seen. Reducing disease burden, including reductions in the more than 200,000 ED visits occurring as a result of gout annually in the US, will require truly innovative approaches that are simultaneously low-cost and transferrable across healthcare systems. Only then can the problem with gout - the fact that it remains such a problem - be effectively overcome.

BRIAN W. COBURN, PhD

Department of Internal Medicine,

Division of Rheumatology,

University of Nebraska Medical Center

and the Veterans Affairs Nebraska-Western Iowa Health Care System, Omaha, Nebraska, USA;

TED R. MIKULS, MD, MSPH,

Umbach Professor of Rheumatology,

Department of Internal Medicine,

Division of Rheumatology,

University of Nebraska Medical Center

and the Veterans Affairs Nebraska-Western Iowa Health Care System,

Omaha, Nebraska, USA

Dr. Coburn has received research support from a University of Nebraska Medical Center Fellowship and the Rheumatology Research Foundation;

Personal non-commercial use only. The Journal of Rheumatology Copyright $\subset$ 2016. All rights reserved. 
Dr. Mikuls received research support from US National Institutes of Health/US National Institute of Arthritis and Musculoskeletal and Skin Diseases (1P50AR060772); US Department of Defense; the Rheumatology Research Foundation; and Astra Zeneca (pending).

Address correspondence to Dr. T.R. Mikuls, University of Nebraska Medical Center, Division of Rheumatology, 983025 Nebraska Medical Center, Omaha, Nebraska 68198,USA; E-mail: tmikuls@unmc.edu

\section{REFERENCES}

1. Zhu Y, Pandya BJ, Choi HK. Prevalence of gout and hyperuricemia in the US general population: the National Health and Nutrition Examination Survey 2007-2008. Arthritis Rheum 2011;63:3136-41.

2. Garg R, Sayles HR, Yu F, Michaud K, Singh J, Saag KG, et al. Gout-related health care utilization in US emergency departments, 2006 through 2008. Arthritis Care Res 2013;65:571-7.

3. Krishnan E, Svendsen K, Neaton JD, Grandits G, Kuller LH. Long-term cardiovascular mortality among middle-aged men with gout. Arch Intern Med 2008;168:1104-10.

4. Wood R, Fermer S, Ramachandran S, Baumgartner S, Morlock R. Patients with gout treated with conventional urate-lowering therapy: association with disease control, health-related quality of life, and work productivity. J Rheumatol (in press).

5. Jinno S, Hasegawa K, Neogi T, Goto T, Dubreuil M. Trends in emergency department visits and charges for gout in the US between 2006 and 2012. J Rheumatol 2016;43:1589-92.

6. Singh JA, Yu S. Time trends, predictors, and outcome of emergency department use for gout: a nationwide US study. J Rheumatol 2016;43:1581-8.

7. Agency for Healthcare Research and Quality. Healthcare Cost and Utilization Project. Introduction to the HCUP Nationwide Emergency Department Sample (NEDS), 2012. [Internet. Accessed June 7, 2016.] Available from: www.hcup-us.ahrq.gov/ db/nation/neds/NEDS_Introduction_2012.jsp

8. Rashid N, Coburn BW, Wu YL, Cheetham TC, Curtis JR, Saag KG, et al. Modifiable factors associated with allopurinol adherence and outcomes among patients with gout in an integrated healthcare system. J Rheumatol 2015;42:504-12.
9. Lynch BA, Van Norman CA, Jacobson RM, Weaver AL, Juhn YJ. Impact of delay in asthma diagnosis on health care service use. Allergy Asthma Proc 2010;31:e48-e52.

10. Oster A, Bindman AB. Emergency department visits for ambulatory care sensitive conditions: insights into preventable hospitalizations. Med Care 2003;41:198-207.

11. Sarawate CA, Brewer KK, Yang W, Patel PA, Schumacher HR, Saag KG, et al. Gout medication treatment patterns and adherence to standards of care from a managed care perspective. Mayo Clin Proc 2006;81:925-34.

12. Singh JA, Hodges JS, Toscano JP, Asch SM. Quality of care for gout in the US needs improvement. Arthritis Rheum 2007;57:822-9.

13. Halpern R, Mody RR, Fuldeore MJ, Patel PA, Mikuls TR. Impact of noncompliance with urate-lowering drug on serum urate and gout-related healthcare costs: administrative claims analysis. Curr Med Res Opin 2009;25:1711-9.

14. Khanna D, Fitzgerald JD, Khanna PP, Bae S, Singh MK, Neogi T, et al. 2012 American College of Rheumatology guidelines for management of gout. Part 1: systematic nonpharmacologic and pharmacologic therapeutic approaches to hyperuricemia. Arthritis Care Res 2012;64:1431-46.

15. Wagner EH, Austin BT, Von Korff M. Organizing care for patients with chronic illness. Milbank Q 1996;74:511-44.

16. Coburn BW, Bendlin KA, Sayles H, Hentzen KS, Hrdy MM, Mikuls TR. Target serum urate: do gout patients know their goal? Arthritis Care Res 2016 Jan 19 (E-pub ahead of print).

17. Rees F, Jenkins W, Doherty M. Patients with gout adhere to curative treatment if informed appropriately: proof-of-concept observational study. Ann Rheum Dis 2013;72:826-30.

18. Witt DM, Sadler MA, Shanahan RL, Mazzoli G, Tillman DJ. Effect of a centralized clinical pharmacy anticoagulation service on the outcomes of anticoagulation therapy. Chest 2005;127:1515-22.

19. Mikuls TR, Cheetham TC, Rashid N, Levy GD, Kerimian A, Low $\mathrm{K}$, et al. A pragmatic cluster-randomized controlled trial of an automated, pharmacy-based intervention to optimize allopurinol therapy in gout [abstract]. Arthritis Rheumatol 2015;67 Suppl:S2590.

J Rheumatol 2016;43:1453-5; doi:10.3899/jrheum.160614 